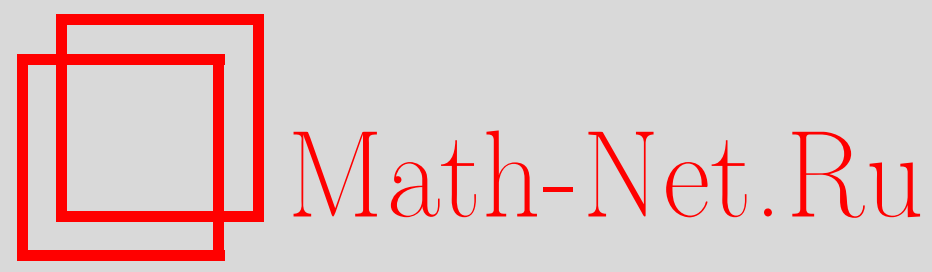

Д. Н. Жук, О классификации автоматных базисов Поста по разрешимости свойств $A$-полноты для дефинитных автоматов, Дискрет. матем., 2010, том 22, выпуск 2, 80-95

DOI: https://doi.org/10.4213/dm1097

Использование Общероссийского математического портала Math-Net.Ru подразумевает, что вы прочитали и согласны с пользовательским соглашением http://www.mathnet.ru/rus/agreement

Параметры загрузки:

IP: 35.173 .219 .12

26 апреля 2023 г., 18:22:23 


\title{
О классификации автоматных базисов Поста по разрешимости свойств $A$-полноты для дефинитных автоматов
}

\author{
() 2010 г. Д. Н. Жук
}

\begin{abstract}
В работе рассматриваются системы вида $M=F \cup v$, где $F$ - некоторый класс Поста, а $v$ - конечная система дефинитных автоматов. Все классы Поста были разделены на те, для которых проблема $A$-полноты таких систем дефинитных автоматов алгоритмически разрешима, и те, для которых проблема $A$-полноты алгоритмически неразрешима.
\end{abstract}

\section{1. Введение}

В работах $[3,4]$ установлена алгоритмическая неразрешимость задач о полноте и $A$-полноте относительно операций суперпозиции и обратной связи для конечных систем автоматных функций. Для систем автоматов, содержащих все булевы функции, указанные задачи алгоритмически разрешимы $[5,6]$. Д. Н. Бабин исследовал на полноту и $A$-полноту системы вида $F \cup v$, где $F$ - некоторый класс Поста, а $v$ - конечная система автоматных функций. Ему удалось построить классификацию классов Поста по их способности гарантировать разрешимость проблемы полноты для конечных систем автоматов. Оказалось, что проблемы полноты и $A$-полноты для систем вида $F \cup v$ разрешимы точно тогда, когда $F$ содержит либо функцию $x+y+z$, либо функцию $x y \vee y z \vee x z$ [7].

Похожие результаты были получены для дефинитных автоматов. Было показано, что в классе дефинитных автоматов задачи о полноте и $A$-полноте относительно операций суперпозиции алгоритмически неразрешимы [8]. Ранее автор показал, что для систем дефинитных автоматов вида $P_{2} \cup v$ существует алгоритм проверки на полноту и $A$-полноту таких систем автоматов [9]. Для каждого конечного $v$ он заключается в проверке непринадлежности $v$ конечному числу предполных классов. Естественно исследовать на $A$-полноту системы вида $F \cup v$, где $F$ - некоторый класс Поста, а $v-$ конечная система дефинитных автоматов. Возникает разделение классов Поста на сильные и слабые по их способности гарантировать разрешимость проблемы $A$-полноты для дефинитных автоматов. В работах $[10,11]$ автором были выделены некоторые сильные и некоторые слабые классы Поста. В данной работе выполнено заключительное построение, которое позволяет все классы Поста разделить на сильные и слабые

Автор благодарен своему научному руководителю В. Б. Кудрявцеву за помощь в исследовании задачи и написании данной работы. 


\section{2. Основные понятия и результаты}

Пусть $\mathbf{N}=\{1,2,3, \ldots\}-$ множество всех натуральных чисел, $\mathbf{N}_{0}=\mathbf{N} \cup\{0\}, E_{2}=\{0,1\}$, $E_{2}^{l}-$ множество всех слов длины $l$ в алфавите $E_{2}, E-$ множество всех бесконечных последовательностей нулей и единиц. Далее такие последовательности называем сверхсловами. Множество $E^{n}$ состоит из всех наборов $\left(\alpha_{1}, \alpha_{2}, \ldots, \alpha_{n}\right)$, где $\alpha_{i} \in E, i=1, \ldots, n$. Если $a, b \in E_{2}$, то $\bar{a}$ - отрицание $a, a \vee b$ - дизъюнкция $a$ и $b, a \& b$ - конъюнкция $a$ и $b, a+b-$ сумма по модулю 2. Множество всех булевых функций будем обозначать через $P_{2}$.

Пусть $\alpha-$ слово или сверхслово. Для $n \in \mathbf{N}$ через $\alpha(n)$ обозначим $n$-й элемент $\alpha$. Обозначим $|\alpha|$ длину слова $\alpha$; для сверхслова $\alpha$ будем полагать, что $|\alpha|=\infty$. Для слова $\alpha$ такого, что $|\alpha| \geqslant k$, определим

$$
\left[{ }_{k} \alpha=\alpha(|\alpha|-k+1) \ldots \alpha(|\alpha|-1) \alpha(|\alpha|) .\right.
$$

Для слова или сверхслова $\alpha$ такого, что $|\alpha| \geqslant k \geqslant l$ положим

$$
\begin{aligned}
]_{k} \alpha & =\alpha(1) \alpha(2) \ldots \alpha(k), \\
{[l]_{k} \alpha } & =\alpha(k-l+1) \alpha(k-l+2) \ldots \alpha(k) .
\end{aligned}
$$

Для произвольного слова $\alpha$ определим слово $\alpha^{s}=\alpha \alpha \ldots \alpha$, составленное из $s$ слов $\alpha$, и сверхслово $\alpha^{\infty}=\alpha \alpha \alpha \ldots$

Пусть $n, h \in \mathbf{N}$. Функция $T: E^{n} \rightarrow E$ называется дефинитным автоматом с $n$ входами высоты $h$, если существуют функции $f_{j}:\left(E_{2}{ }^{j}\right)^{n} \rightarrow E_{2}, j=1,2,3, \ldots, h$, такие, что для любых $x_{1}, x_{2}, \ldots, x_{n} \in E$

$$
\begin{aligned}
T\left(x_{1}, x_{2}, \ldots, x_{n}\right)(1) & \left.\left.\left.=f_{1}(]_{1} x_{1},\right]_{1} x_{2}, \ldots,\right]_{1} x_{n}\right), \\
T\left(x_{1}, x_{2}, \ldots, x_{n}\right)(2) & \left.\left.\left.=f_{2}(]_{2} x_{1},\right]_{2} x_{2}, \ldots,\right]_{2} x_{n}\right), \\
& \ldots \\
T\left(x_{1}, x_{2}, \ldots, x_{n}\right)(h) & \left.\left.\left.=f_{h}(]_{h} x_{1},\right]_{h} x_{2}, \ldots,\right]_{h} x_{n}\right), \\
T\left(x_{1}, x_{2}, \ldots, x_{n}\right)(h+1) & =f_{h}\left([h]_{h+1} x_{1},[h]_{h+1} x_{2}, \ldots,[h]_{h+1} x_{n}\right), \\
& \ldots \\
T\left(x_{1}, x_{2}, \ldots, x_{n}\right)(h+i) & =f_{h}\left([h]_{h+i} x_{1},[h]_{h+i} x_{2}, \ldots,[h]_{h+i} x_{n}\right),
\end{aligned}
$$

Таким образом, согласно нашему определению автомат высоты $h$ является также автоматом высоты $h+1$. Элемент $T\left(x_{1}, x_{2}, \ldots, x_{n}\right)(j)$ будем называть элементом на выходе автомата $T$ в момент времени $j$, а $x_{i}(j)$ - элементом, подаваемым на $i$-й вход в момент времени $j$. Для $j=1, \ldots, h-1$ функция $f_{j}$ определяет элемент на выходе автомата $T$ в момент времени $j$, а функция $f_{h}$ определяет элемент на выходе автомата, начиная с момента времени $h$.

Пусть $T$ - автомат высоты $h$. Для $p \in \mathbf{N}$ определим функцию $T^{p}:\left(E_{2}{ }^{p}\right)^{n} \rightarrow E_{2}$. Если $p \leqslant h$, то положим

$$
T^{p}\left(\alpha_{1}, \alpha_{2}, \ldots, \alpha_{n}\right)=f_{p}\left(\alpha_{1}, \alpha_{2}, \ldots, \alpha_{n}\right)
$$

Для $p>h$ положим

$$
T^{p}\left(\alpha_{1}, \alpha_{2}, \ldots, \alpha_{n}\right)=f_{h}\left(\left[{ }_{h} \alpha_{1},\left[{ }_{h} \alpha_{2}, \ldots,\left[{ }_{h} \alpha_{n}\right) .\right.\right.\right.
$$


Таким образом, для любого $p$ функция $T^{p}$ определяет элемент на выходе автомата $T$ в момент времени $p$. Функции $T^{p}$, где $p=1, \ldots, h$, будем называть порождающими. Нетрудно убедиться, что для задания дефинитного автомата необходимо задать высоту автомата и порождающие функции.

Множество всех дефинитных автоматов обозначим $\mathscr{P}_{a}$. Для $T \in \mathscr{P}_{a}$ через $h(T)$ обозначим наименьшую высоту автомата $T$. Ясно, что каждой булевой функции из $P_{2}$ соответствует дефинитный автомат высоты 1 . Будем использовать стандартные обозначения для автоматов высоты 1 , а именно: $\bar{x}, x \& y=x y, x \vee y, x+y$ - дефинитные автоматы $T_{1}, T_{2}, T_{3}$ и $T_{4}$ высоты 1 такие, что выполняются равенства

$$
\begin{aligned}
T_{1}^{1}(\alpha) & =\bar{\alpha}, & & T_{2}^{1}(\alpha, \beta)=\alpha \& \beta, \\
T_{3}^{1}(\alpha, \beta) & =\alpha \vee \beta, & & T_{4}^{1}(\alpha, \beta)=\alpha+\beta .
\end{aligned}
$$

Пусть $S_{c}$ - автомат высоты 2 с одним входом, для которого $S_{c}^{1}(\alpha)=c, S_{c}^{2}(\alpha)=\alpha(1)$. Автомат $S_{c}$ будем называть задержкой с начальным состоянием $c$.

Пусть $M \subseteq \mathscr{P}_{a}$. Фиксируем некоторое счетное множество $U=\left\{u_{1}, u_{2}, u_{3}, \ldots\right\}$, элементы которого будем называть переменными. Индуктивно определим понятие терма над множеством $M$ :

(1) если $u \in U$, то $u$ - терм над $M$,

(2) если $F$ - автомат с $n \in \mathbf{N}$ входами, $F \in M, \tau_{1}, \tau_{2}, \ldots, \tau_{n}-$ термы над $M$, то выражение $F\left(\tau_{1}, \tau_{2}, \ldots, \tau_{n}\right)$ - терм над $M$.

Термы, отличные от переменных, назовем собственными. Пусть $\tau$ - произвольный терм, $\left(x_{1}, x_{2}, \ldots, x_{m}\right)$ - набор попарно различных переменных, содержащий все переменные, использованные при построении терма $\tau$. Тогда через $\tau\left(x_{1}, x_{2}, \ldots, x_{m}\right)$ обозначим функцию $\tau: E^{m} \rightarrow E$, определяемую индуктивно:

(1) если $\tau=x_{c}$ - переменная, $\gamma=\left(\gamma^{1}, \gamma^{2}, \ldots, \gamma^{m}\right) \in E^{m}$, то определим

$$
\tau\left(x_{1}, x_{2}, \ldots, x_{m}\right)(\gamma)=\gamma^{c},
$$

(2) если $\tau=F\left(\tau_{1}, \tau_{2}, \ldots, \tau_{n}\right), \gamma=\left(\gamma^{1}, \gamma^{2}, \ldots, \gamma^{m}\right) \in E^{m}$, то определим

$$
\tau\left(x_{1}, x_{2}, \ldots, x_{m}\right)(\gamma)=F\left(\tau_{1}\left(x_{1}, x_{2}, \ldots, x_{m}\right)(\gamma), \ldots, \tau_{n}\left(x_{1}, x_{2}, \ldots, x_{m}\right)(\gamma)\right) .
$$

О функции $T$ такой, что $T=\tau\left(x_{1}, x_{2}, \ldots, x_{m}\right)$ для некоторого собственного терма $\tau$ над множеством $M$, будем говорить, что она получена термальными операциями из дефинитных автоматов множества $M$. Нетрудно проверить, что функция $T$ также будет дефинитным автоматом, поэтому мы можем ввести на множестве $\mathscr{P}_{a}$ оператор замыкания [·] относительно термальных операций - такое отображение, которое каждому множеству $M \subseteq \mathscr{P}_{a}$ сопоставляет множество $[M]$ всех автоматов, которые можно получить термальными операциями из автоматов множества $M$. Определенный выше оператор замыкания также известен как оператор замыкания относительно операций суперпозиции [12].

Множество $M$ называется замкнутым, если $[M]=M$; множество $M$ называется полным, если $[M]=\mathscr{P}_{a}$. Пусть $\tau \in \mathbf{N}$, скажем, что автоматы $T_{1}$ и $T_{2}$ с $n$ входами $\tau$-равны, если для любого $i \leqslant \tau$ справедливо равенство $T_{1}^{i}=T_{2}^{i}$. Обозначим через $[M]_{\tau}$ множество всех дефинитных автоматов, $\tau$-равных получающимся из $M$ с помощью термальных операций. Множество $M$ называется $A$-полным, если $[M]_{\tau}=\mathscr{P}_{a}$ для любого $\tau$. Положим

$$
[M]_{A}=\bigcap_{\tau=1}^{\infty}[M]_{\tau} .
$$


Проблема $A$-полноты для $\mathscr{P}_{a}$ состоит в описании всех $A$-полных множеств $M$. Множество $M$ называется $A$-замкнутым, если $[M]_{A}=M ; M$ называется $A$-предполным, если $[M]_{A} \neq \mathscr{P}_{a}$ и для любой $f \in \mathscr{P}_{a} \backslash M$ справедливо равенство

$$
[M \cup\{f\}]_{A}=\mathscr{P}_{a} .
$$

Нетрудно заметить, что дефинитные автоматы - это все автоматы, которые можно получить с помощью термальных операций из автоматов из $P_{2}$ и задержки. Другими словами $\left[P_{2} \cup\left\{S_{c}\right\}\right]=\mathscr{P}_{a}$, где $S_{c}-$ задержка с начальным состоянием $c$. Отсюда, в частности, следует, что $\left[\left\{\bar{x} \vee \bar{y}, S_{c}\right\}\right]=\mathscr{P}_{a}$.

Постом полностью описаны все замкнутые относительно операций суперпозиции классы булевых функций $[1,2]$. Все они конечнопорожденные и образуют счетную решетку по включению.

Ранее автор показал, что для систем дефинитных автоматов вида $P_{2} \cup v$ существует алгоритм проверки на полноту и $A$-полноту таких систем дефинитных автоматов [9]. Значит, для распознавания полноты и $A$-полноты существенна роль функций без памяти, присутствующих в базисе. Если присутствуют все функции без памяти, то проблемы $A$-полноты и полноты для дефинитных автоматов алгоритмически разрешимы. Если присутствует, фактически, лишь тождественная функция $x$, то не существует алгоритма распознавания $A$-полноты и полноты для дефинитных автоматов $[4,8]$.

Учитывая эти результаты, естественно исследовать на $A$-полноту и полноту системы вида $F \cup v$, где $F$ - некоторый класс Поста, а $v$ - конечная система дефинитных автоматов. Такие системы мы будем называть автоматными базисами Поста - так же, как это делал Д. Н. Бабин в работах $[5,7]$.

Пусть $F-$ замкнутый класс булевых функций. Определим проблему $A$-ПолнОТА $(F)$ следующим образом: дана конечная система $v$ дефинитных автоматов, заданных своими порождающими функциями; требуется установить, $A$-полна ли система $F \cup v$.

Нетрудно убедиться, что если $F \subseteq F^{\prime}$ и проблема $A$-ПолнотА $(F)$ алгоритмически разрешима, то $A$-ПолнотА $\left(F^{\prime}\right)$ также алгоритмически разрешима. Аналогично, если $F^{\prime} \subseteq F$ и проблема $A$-ПолнотА $(F)$ алгоритмически неразрешима, то $A$-ПолнотА $\left(F^{\prime}\right)$ также алгоритмически неразрешима. Также, если $F^{*}$ - класс, двойственный к $F$ относительно замены 0 на 1 , то проблема $A$-ПолнотА $\left(F^{*}\right)$ алгоритмически разрешима тогда и только тогда, когда алгоритмически разрешима проблема $A$-ПолнотА $(F)$.

Воспользуемся обозначениями из [1]. Для $\mu \geqslant 2$

$$
h_{\mu}\left(x_{1}, x_{2}, \ldots, x_{\mu+1}\right)=\bigvee_{i=1}^{\mu+1} x_{1} x_{2} \ldots x_{i-1} x_{i+1} \ldots x_{\mu+1},
$$

$h_{\mu}^{*}-$ функция, двойственная к $h_{\mu}$. В частности,

$$
h_{2}\left(x_{1}, x_{2}, x_{3}\right)=x_{1} x_{2} \vee x_{2} x_{3} \vee x_{1} x_{3}
$$

Будем использовать также обозначения

$$
\begin{aligned}
& F_{2}^{3}=\left[\left\{h_{3}^{*}\right\}\right], \quad F_{6}^{3}=\left[\left\{h_{3}\right\}\right], \quad D_{2}=\left[\left\{h_{2}\right\}\right], \quad L_{4}=[\{x+y+z\}], \\
& F_{4}^{\infty}=[\{x \vee \bar{y}\}], \quad F_{8}^{\infty}=[\{x \& \bar{y}\}], \quad S_{6}=[\{x \vee y, 0,1\}], \quad P_{6}=[\{x \& y, 0,1\}], \\
& O_{9}=[\{\bar{x}, 0\}], \quad F_{4}^{4}=\left[\left\{x \vee \bar{y}, h_{4}^{*}\right\}\right], \quad F_{8}^{4}=\left[\left\{x \& \bar{y}, h_{4}\right\}\right] .
\end{aligned}
$$

Ранее автором были доказаны две следующие теоремы (соответственно в [10] и [11]). 
Теорема 1 ([10]). Проблема $A$-ПолнОтА $(F)$ алгоритмически неразрешима для каждого $F \in\left\{F_{4}^{\infty}, F_{8}^{\infty}, S_{6}, P_{6}, O_{9}\right\}$.

Теорема 2 ([11]). Проблема $A$-ПолнотА $(F)$ алгоритмически разрешима для каждого $F \in\left\{F_{2}^{3}, F_{6}^{3}, D_{2}, L_{4}\right\}$.

В данной работе доказывается следующая теорема.

Теорема 3. Проблема $A$-ПолНОТА $(F)$ алгоритмически неразрешима для каждого $F \in\left\{F_{4}^{4}, F_{8}^{4}\right\}$.

Эта теорема завершает построение классификации классов Поста по их способности гарантировать разрешимость проблемы $A$-полноты для дефинитных автоматов. Таким образом, справедливо следующее утверждение.

Теорема 4. Проблема $A$-ПолНОтА $(F)$ алгоритмически разрешима тогда и только тогда, когда для какой-то $f \in\left\{h_{2}, x+y+z, h_{3}, h_{3}^{*}\right\}$ справедливо включение $f \in F$.

\section{3. Основные утверждения}

Тройка $\theta=\langle D, \rho, \omega\rangle$, где $D=\left\{d_{1}, d_{2}, \ldots, d_{k}\right\}, D^{*}-$ множество слов в алфавите $D$, $\rho: D \rightarrow D^{*}, \rho\left(d_{i}\right)=R_{i}, \omega-$ натуральное число, называется системой однородных продукций Поста. Если $\xi \in D^{*},|\xi| \geqslant \omega$, то скажем, что $\theta$ применима к слову $\xi=g_{1} g_{2} \ldots g_{|\xi|}$, где $g_{1}=d_{c}$, и слово $\theta(\xi)=g_{\omega+1} g_{\omega+2} \ldots g_{|\xi|} R_{c}$ назовем результатом применения $\theta$ к слову $\xi$. Последовательность $\xi_{1}, \xi_{2}, \xi_{3}, \ldots$ такую, что $\xi_{1}=\xi$, а $\xi_{i+1}=\theta\left(\xi_{i}\right)$, назовем последовательностью продукций Поста слова $\xi$. Известно [13], что существует система однородных продукций Поста, для которой не существует алгоритма, по слову $\xi$ решающего вопрос о конечности последовательности продукций слова $\xi$. Зафиксируем эту систему продукций Поста $\langle D, \rho, \omega\rangle$.

Для определенности будем считать, что $k$ - четное число. Если это не так, добавим в $D$ еще один элемент и определим произвольным образом $\rho\left(d_{k+1}\right)$. Понятно, что при этом проблема конечности последовательности продукций Поста останется неразрешимой. Для $g=d_{i} \in D$ определим $\tilde{g}=1^{i} 01^{k+1-i}$. Введем обозначения

$$
\begin{array}{lll}
e_{1}=(01)^{5} 00010001(01)^{k / 2+1}, & e_{2}=(10)^{5} 10100000(10)^{k / 2+1}, \\
p_{0}=1(01) 1(10) 1(10) 1(1)^{8}, & p_{1}=1(10) 1(00) 1(10) 1(1)^{8}, \\
p_{2}=1(10) 1(00) 1(00) 1(1)^{8}, & p_{3}=1(10) 1(10) 1(10) 1(1)^{8} .
\end{array}
$$

Нетрудно убедиться, что для любых $i, j$ и $l$ выполняется равенство

$$
\left|p_{i}\right|+\left|\tilde{d}_{j}\right|=\left|e_{l}\right|=k+20 .
$$

В процессе доказательства все сверхслова будут разбиваться на слова длины $k+20$. При этом будут встречаться слова вида $p_{i} \tilde{d}_{j}$ и $e_{i}$. Первые десять символов в слове $p_{i} \tilde{d}_{j}$ являются заголовком и задают $i$. Следующие восемь символов используются только в словах $e_{1}$ и $e_{2}$ и служат для определения остатка от деления номера позиции на $k+20$. И наконец, последние $k+2$ символа задают $j$ в словах вида $p_{i} \tilde{d}_{j}$.

Пусть $g_{i} \in D$ для любого $i, m_{1}<m_{2}<m_{3}$. Сверхслова вида

$$
\begin{aligned}
& p_{1} \tilde{g}_{1} p_{1} \tilde{g}_{2} \ldots p_{1} \tilde{g}_{m_{1}} p_{2} \tilde{g}_{m_{1}+1} p_{3} \tilde{g}_{m_{1}+2} \ldots p_{3} \tilde{g}_{m_{2}} p_{0} \tilde{g}_{m_{2}+1} p_{0} \tilde{g}_{m_{2}+2} \ldots, \\
& \quad p_{1} \tilde{g}_{1} p_{1} \tilde{g}_{2} \ldots p_{1} \tilde{g}_{m_{1}} p_{2} \tilde{g}_{m_{1}+1} p_{3} \tilde{g}_{m_{1}+2} \ldots p_{3} \tilde{g}_{m_{2}} p_{0} \tilde{g}_{m_{2}+1} p_{0} \tilde{g}_{m_{2}+2} \ldots p_{0} \tilde{g}_{m_{3}} 1^{\infty}
\end{aligned}
$$


будем называть записями продукции $g_{m_{1}+1} g_{m_{1}+2} \ldots g_{m_{2}}$.

Пусть

$$
C_{01}=\left\{(01)^{n} 1^{\infty} \mid n \in \mathbf{N}_{0}\right\} .
$$

Определим отображение $\widetilde{H}_{01}: C_{01} \rightarrow E$ такое, что

$$
\tilde{H}_{01}\left((01)^{n} 1^{\infty}\right)=(01)^{n+1} 1^{\infty} \text {. }
$$

Пусть

$$
C_{10}=\left\{(10)^{n} 1^{\infty} \mid n \in \mathbf{N}_{0}\right\} .
$$

Определим отображение $\tilde{H}_{10}: C_{10} \rightarrow E$ такое, что

$$
\tilde{H}_{10}\left((10)^{n} 1^{\infty}\right)=(10)^{n+1} 1^{\infty} \text {. }
$$

Пусть $V_{e}-$ множество пар вида $\left(\left(e_{1}\right)^{n}(01)^{\infty},\left(e_{2}\right)^{n}(10)^{\infty}\right)$, где $n \in \mathbf{N}_{0}$. Определим отображения $\tilde{H}_{1}: V_{e} \rightarrow E$ и $\tilde{H}_{2}: V_{e} \rightarrow E$ такие, что

$$
\begin{aligned}
& \tilde{H}_{1}\left(\left(e_{1}\right)^{n}(01)^{\infty},\left(e_{2}\right)^{n}(10)^{\infty}\right)=\left(e_{1}\right)^{n+1}(01)^{\infty}, \\
& \widetilde{H}_{2}\left(\left(e_{1}\right)^{n}(01)^{\infty},\left(e_{2}\right)^{n}(10)^{\infty}\right)=\left(e_{2}\right)^{n+1}(10)^{\infty} .
\end{aligned}
$$

Пусть $C_{F}-$ множество сверхслов вида $p_{0} \tilde{g}_{1} p_{0} \tilde{g}_{2} \ldots p_{0} \tilde{g}_{n} 1^{\infty}$, где $n \in \mathbf{N}_{0}, g_{i} \in D$ для любого $i$. Для $d_{c} \in D$ определим $\widetilde{F}_{c}: C_{F} \rightarrow E$ такое, что

$$
\tilde{F}_{c}\left(p_{0} \tilde{g}_{1} p_{0} \tilde{g}_{2} \ldots p_{0} \tilde{g}_{n} 1^{\infty}\right)=p_{0} \tilde{g}_{1} p_{0} \tilde{g}_{2} \ldots p_{0} \tilde{g}_{n} p_{0} \tilde{d}_{c} 1^{\infty} \text {. }
$$

Пусть $d_{c} \in D, V_{c}-$ множество троек вида $\left(p_{1} \tilde{g}_{1} p_{1} \tilde{g}_{2} \ldots p_{1} \tilde{g}_{m_{1}} p_{2} \tilde{g}_{m_{1}+1} p_{3} \tilde{g}_{m_{1}+2} \ldots\right.$ $\left.p_{3} \tilde{g}_{m_{2}} p_{0} \tilde{g}_{m_{2}+1} p_{0} \tilde{g}_{m_{2}+2} \ldots, e_{1}^{\infty}, e_{2}^{\infty}\right)$, где $g_{i} \in D$ для любого $i, g_{m_{1}+1}=d_{c}, m_{2}-m_{1} \geqslant \omega$ и $g_{m_{2}+1} g_{m_{2}+2} \ldots g_{m_{2}+\left|R_{c}\right|}=R_{c}$. Определим $\widetilde{T}_{c}: V_{c} \rightarrow E$, полагая

$$
\widetilde{T}_{c}\left(p_{1} \tilde{g}_{1} p_{1} \tilde{g}_{2} \ldots p_{1} \tilde{g}_{m_{1}} p_{2} \tilde{g}_{m_{1}+1} p_{3} \tilde{g}_{m_{1}+2} \ldots p_{3} \tilde{g}_{m_{2}} p_{0} \tilde{g}_{m_{2}+1} p_{0} \tilde{g}_{m_{2}+2} \ldots, e_{1}^{\infty}, e_{2}^{\infty}\right)=\beta,
$$

где

$$
\begin{aligned}
\beta=p_{1} \tilde{g}_{1} p_{1} \tilde{g}_{2} \ldots p_{1} \tilde{g}_{m_{1}+\omega} p_{2} \tilde{g}_{m_{1}+\omega+1} p_{3} \tilde{g}_{m_{1}+\omega+2} \ldots & \\
& p_{3} \tilde{g}_{m_{2}+\left|R_{c}\right|} p_{0} \tilde{g}_{m_{2}+\left|R_{c}\right|+1} p_{0} \tilde{g}_{m_{2}+\left|R_{c}\right|+2} \ldots
\end{aligned}
$$

Другими словами, отображение $\widetilde{T}_{c}$ преобразует запись продукции $\xi$ в запись продукции $\theta(\xi)$. Стоит отметить, что запись продукции $\theta(\xi)$ уже содержится в сверхслове, подаваемом на первый вход. Отображение $\widetilde{T}_{c}$ лишь преобразует заголовки $p_{0}, p_{1}, p_{2}$ и $p_{3}$.

Пусть $V_{F}$ - множество троек вида $\left(p_{1} \tilde{g}_{1} p_{1} \tilde{g}_{2} p_{1} \tilde{g}_{3} \ldots, e_{1}^{\infty}, e_{2}^{\infty}\right)$, где $g_{i} \in D$ для любого $i$. Определим $\widetilde{F}: V_{F} \rightarrow E$, полагая

$$
\widetilde{F}\left(p_{1} \tilde{g}_{1} p_{1} \tilde{g}_{2} p_{1} \tilde{g}_{3} \ldots, e_{1}^{\infty}, e_{2}^{\infty}\right)=0^{\infty} .
$$

Для произвольного слова $\xi=g_{1} g_{2} \ldots g_{l} \in D^{*}$ определим множество $C_{\xi}$ сверхслов вида $p_{0} \tilde{g}_{1} p_{0} \tilde{g}_{2} \ldots p_{0} \tilde{g}_{l} p_{0} \tilde{g}_{l+1} p_{0} \tilde{g}_{l+2} \ldots p_{0} \tilde{g}_{n} 1^{\infty}$, где $g_{l+1}, g_{l+2}, \ldots, g_{n} \in D$. Также определим отображение $\widetilde{T}_{\xi}: C_{\xi} \rightarrow E$ такое, что

$$
\begin{aligned}
\tilde{T}_{\xi}\left(p_{0} \tilde{g}_{1} p_{0} \tilde{g}_{2} \ldots p_{0} \tilde{g}_{l} p_{0} \tilde{g}_{l+1} p_{0} \tilde{g}_{l+2} \ldots p_{0} \tilde{g}_{n} 1^{\infty}\right) \\
\quad=p_{2} \tilde{g}_{1} p_{3} \tilde{g}_{2} \ldots p_{3} \tilde{g}_{l} p_{0} \tilde{g}_{l+1} p_{0} \tilde{g}_{l+2} \ldots p_{0} \tilde{g}_{n} 1^{\infty} .
\end{aligned}
$$


Другими словами, на выходе отображения $\widetilde{T}_{\xi}$ будет запись продукции $\xi$.

Таким образом, отображение $\widetilde{H}_{01}$ определено на $C_{01}, \widetilde{H}_{10}$ определено на $C_{10}, \widetilde{H}_{1}$ и $\widetilde{H}_{2}$ на $V_{e}, \widetilde{F}_{c}$ на $C_{F}, \widetilde{T}_{c}$ на $V_{c}, \widetilde{F}$ на $V_{F}, \widetilde{T}_{\xi}$ на $C_{\xi}$.

Построим дефинитные автоматы $H_{01}, H_{10}, H_{1}, H_{2}, F_{1}, \ldots, F_{k}, T_{1}, \ldots, T_{k}, F, T_{\xi}$, соответствующие этим отображениям. Определим высоты автоматов. Положим

$$
\begin{aligned}
h\left(H_{01}\right) & =3, & h\left(H_{10}\right) & =3, \\
h\left(H_{1}\right) & =(k+20) \cdot 2, & h\left(H_{2}\right) & =(k+20) \cdot 2, \\
h\left(F_{c}\right) & =(k+20) \cdot 2, & h(F) & =(k+20) \cdot 2, \\
h\left(T_{c}\right) & =\left(\omega+\left|R_{c}\right|+2\right)(k+20), & h\left(T_{\xi}\right) & =(|\xi|+2)(k+20) .
\end{aligned}
$$

Определим сразу все перечисленные автоматы. Пусть $H \in\left\{H_{01}, H_{10}, H_{1}, H_{2}\right.$, $\left.F_{1}, \ldots, F_{k}, T_{1}, \ldots, T_{k}, F, T_{\xi}\right\}$, автомату $H$ соответствует отображение $\widetilde{H}$, которое определено на множестве $C$. Зададим порождающие функции автомата $H$.

Для $i<h(H)$ положим $H^{i}(r)=0$ тогда и только тогда, когда существует $r^{\prime} \in C$ такое, что $]_{i} r^{\prime}=r$ и для сверхслова $\beta=\widetilde{H}\left(r^{\prime}\right)$ выполняется равенство $\beta(i)=0$. Аналогично $H^{h(H)}(r)=0$ тогда и только тогда, когда существуют $l \geqslant h(H)$ и $r^{\prime} \in C$ такие, что $[h(H)]_{l} r^{\prime}=r$ и для сверхслова $\beta=\widetilde{H}\left(r^{\prime}\right)$ выполняется равенство $\beta(l)=0$.

Определение 1. Дефинитный автомат $T \mathrm{c} n$ входами называется автоматным доопределением отображения $\widetilde{T}: C \rightarrow E$, если $C \subseteq E^{n}$ и для любого $r \in C$ выполняется равенство $T(r)=\widetilde{T}(r)$.

Предложение 1. Дефинитные автоматы $H_{01}, H_{10}, H_{1}, H_{2}, F_{1}, \ldots, F_{k}, T_{1}, \ldots, T_{k}, F, T_{\xi}$ являются автоматныли доопределениями отображении $\widetilde{H}_{01}, \widetilde{H}_{10}, \widetilde{H}_{1}, \widetilde{H}_{2}, \widetilde{F}_{1}, \ldots, \widetilde{F}_{k}$, $\widetilde{T}_{1}, \ldots, \widetilde{T}_{k}, \widetilde{F}, \widetilde{T}_{\xi}$ соответственно.

Для каждого слова $\xi$ такого, что последовательность продукций слова $\xi$ конечна и имеет вид $\xi=\xi_{1}, \xi_{2}, \ldots, \xi_{s}$, определим множество $W_{\xi} \subseteq \mathbf{N}^{3}$. Пусть для любого $t$ слово $\xi_{t}$ начинается с буквы $d_{i_{t}}$. Рассмотрим две последовательности натуральных чисел:

$$
a_{i}=(i-1) \omega, \quad b_{i}=a_{i}+\left|\xi_{i}\right| .
$$

Положим

$$
n_{4}(\xi)=b_{s}(k+20)+6, \quad \xi_{1} R_{i_{1}} R_{i_{2}} \ldots R_{i_{s-1}}=g_{1} g_{2} \ldots g_{l} .
$$

Для $1 \leqslant i \leqslant s$ положим

$$
\zeta_{i}=p_{1} \tilde{g}_{1} p_{1} \tilde{g}_{2} \ldots p_{1} \tilde{g}_{a_{i}} p_{2} \tilde{g}_{a_{i}+1} p_{3} \tilde{g}_{a_{i}+2} \ldots p_{3} \tilde{g}_{b_{i}} p_{0} \tilde{g}_{b_{i}+1},
$$

то есть $\zeta_{i}$ - начало записи продукции $\xi_{i}$.

Тройка $\left(n_{1}, n_{2}, n_{3}\right)$ принадлежит $W_{\xi}$, если и только если $n_{1}, n_{2}, n_{3} \leqslant n_{4}(\xi)$ и числа $n_{1}, n_{2}, n_{3}$ удовлетворяют одному из следующих условий:

(1) $n_{1}=n_{4}(\xi)-1 ; n_{2}=n_{3}=n_{4}(\xi)$;

(2) $n_{1}=(k+20) n+q_{1}, n_{2}=(k+20) n+q_{2}$, где $n \in \mathbf{N}_{0}, 0 \leqslant q_{1}, q_{2} \leqslant(k+20), q_{2}-q_{1}=1$ и $\left(q_{1}, q_{2}\right) \notin\{(5,6),(8,9),(11,12),(12,13),(14,15),(15,16),(16,17),(17,18)\}$; $n_{3}=n_{4}(\xi)$.

(3) $n_{1}=(k+20) n+8 ; n_{2}=(k+20) n+9 ; n \neq a_{i}$ для любого $i ; n_{3}=n_{4}(\xi)$; 
(4) $n_{1}=(k+20) n+8 ; n_{2}=(k+20) n+9$; существует $i$ такое, что $n=a_{i}, \zeta_{i}\left(n_{3}\right)=1$, $n_{3} \leqslant b_{i}(k+20)+4$.

Нетрудно убедиться, что для любой тройки $\left(n_{1}, n_{2}, n_{3}\right) \in W_{\xi}$ справедливо равенство $n_{1}+1=n_{2}$.

Определение 2. Говорим, что автомат $T \in P_{a}$ с $n$ входами сохраняет $C \subseteq E$, если для любых $\alpha_{1}, \alpha_{2}, \ldots, \alpha_{n} \in C$ выполняется включение $T\left(\alpha_{1}, \alpha_{2}, \ldots, \alpha_{n}\right) \in C$.

Нетрудно убедиться, что справедливо следующее утверждение.

Предложение 2. Для любого $C \subseteq E$ множество всех автоматов, сохраняюших множество С, замкнуто.

По $n_{4}(\xi)$ и множеству $W_{\xi}$ построим множество сверхслов $\widetilde{W}_{\xi}$. Сверхслово $\alpha$ принадлежит $\widetilde{W}_{\xi}$ тогда и только тогда, когда $\alpha\left(n_{1}\right) \vee \alpha\left(n_{2}\right) \vee \alpha\left(n_{3}\right) \vee \alpha\left(n_{4}(\xi)\right)=1$ для любого $\left(n_{1}, n_{2}, n_{3}\right) \in W_{\xi}$. Из предложения 2 следует, что класс автоматов, сохраняющих $\widetilde{W}_{\xi}$, замкнут.

Предложение 3. Каждый из автоматов $x \vee \bar{y}, x \vee S_{0}(y), h_{4}^{*}, H_{01}, H_{10}, H_{1}, H_{2}, F_{1}, \ldots, F_{k}$, $F, T_{1}, \ldots, T_{k}, T_{\xi}$ сохраняет множество $\widetilde{W}_{\xi}$ для любого $\xi$ такого, что последовательность продукций Поста слова $\xi$ конечна.

Предложение 4. Пусть последовательность продукций слова छ бесконечна, тогда система

$$
\Sigma=\left\{x \vee \bar{y}, x \vee S_{0}(y), H_{01}, H_{10}, H_{1}, H_{2}, F_{1}, \ldots, F_{k}, T_{1}, \ldots, T_{k}, F, T_{\xi}\right\}
$$

А-полна.

Доказательство. Пусть последовательность продукций Поста слова $\xi$ бесконечна и имеет вид $\xi_{1}, \xi_{2}, \xi_{3}, \ldots$ Также пусть для любого $t$ слово $\xi_{t}$ начинается с буквы $d_{i_{t}}$.

Так как $x \vee \bar{x}=1$ для любого $x$, константа 1 принадлежит $[\Sigma]$. Пусть

$$
\tau=s \omega(k+20), \quad s \in \mathbf{N} .
$$

Положим

$$
\begin{aligned}
& Q_{01}(x)=H_{01}\left(H_{01}\left(H_{01}\left(\ldots\left(H_{01}(x)\right) \ldots\right)\right)\right), \\
& Q_{10}(x)=H_{10}\left(H_{10}\left(H_{10}\left(\ldots\left(H_{10}(x)\right) \ldots\right)\right)\right),
\end{aligned}
$$

где символы $H_{01}$ и $H_{10}$ входят в формулу $\tau$ раз.

Нетрудно убедиться, что первые $\tau$ символов сверхслова $Q_{01}\left(1^{\infty}\right)$ совпадают с первыми $\tau$ символами сверхслова $(01)^{\infty}$, а первые $\tau$ символов сверхслова $Q_{10}\left(1^{\infty}\right)$ совпадают с первыми $\tau$ символами сверхслова $(10)^{\infty}$.

Определим рекурсивно две последовательности сверхслов. Пусть

$$
\begin{array}{ll}
\beta_{0}=(01)^{\infty}, & \gamma_{0}=(10)^{\infty}, \\
\beta_{i}=H_{1}\left(\beta_{i-1}, \gamma_{i-1}\right), & \gamma_{i}=H_{2}\left(\beta_{i-1}, \gamma_{i-1}\right) .
\end{array}
$$

Нетрудно убедиться, что для любого $i$

$$
\beta_{i}=\left(e_{1}\right)^{i}(01)^{\infty}, \quad \gamma_{i}=\left(e_{2}\right)^{i}(10)^{\infty}
$$


Значит, существуют автоматы $G_{1}, G_{2} \in[\Sigma]$ такие, что

$$
\begin{aligned}
& ]_{\tau} G_{1}\left((01)^{\infty},(10)^{\infty}\right)=\left(e_{1}\right)^{s \omega}, \\
& ]_{\tau} G_{2}\left((01)^{\infty},(10)^{\infty}\right)=\left(e_{2}\right)^{s \omega} .
\end{aligned}
$$

Определим автоматы $L_{1}, L_{2} \in[\Sigma]$ следующим образом:

$$
\begin{aligned}
& L_{1}(x)=G_{1}\left(Q_{01}(x \vee \bar{x}), Q_{10}(x \vee \bar{x})\right), \\
& L_{2}(x)=G_{2}\left(Q_{01}(x \vee \bar{x}), Q_{10}(x \vee \bar{x})\right) .
\end{aligned}
$$

Рассмотрим слово $\xi_{1} R_{i_{1}} R_{i_{2}} \ldots R_{i_{s-1}} R_{i_{s}}=d_{j_{1}} d_{j_{2}} \ldots d_{j_{l}}$. Очевидно, что $l \geqslant s \omega$. Введем обозначение

$$
G_{3}(x)=F_{j_{l}}\left(F_{j_{l-1}}\left(\ldots\left(F_{j_{2}}\left(F_{j_{1}}(x)\right)\right) \ldots\right)\right)
$$

Легко проверить, что

$$
G_{3}\left(1^{\infty}\right)=F_{j_{l}}\left(F_{j_{l-1}}\left(\ldots\left(F_{j_{2}}\left(F_{j_{1}}\left(1^{\infty}\right)\right)\right) \ldots\right)\right)=p_{0} \tilde{d}_{j_{1}} p_{0} \tilde{d}_{j_{2}} \ldots p_{0} \tilde{d}_{j_{l}} 1^{\infty}
$$

Пусть

$$
\delta_{1}=T_{\xi}\left(p_{0} \tilde{d}_{j_{1}} p_{0} \tilde{d}_{j_{2}} \ldots p_{0} \tilde{d}_{j_{l}} 1^{\infty}\right)
$$

Легко проверить, что $\delta_{1}$ является записью продукции $\xi$. Определим индуктивно

$$
\delta_{r+1}=T_{i_{r}}\left(\delta_{r},\left(e_{1}\right)^{\infty},\left(e_{2}\right)^{\infty}\right) .
$$

Тогда существует автомат $G_{0} \in[\Sigma]$ такой, что $G_{0}\left(\delta_{1},\left(e_{1}\right)^{\infty},\left(e_{2}\right)^{\infty}\right)=\delta_{s+1}$. Сверхслово $\delta_{r}$ является записью продукции $\xi_{r}$ для $r=1,2, \ldots, s, s+1$. Первые $\tau$ символов сверхслова $F\left(\delta_{s+1}\right)$ равны нулю.

Таким образом, получен автомат $F\left(G_{0}\left(T_{\xi}\left(G_{3}(x \vee \bar{x})\right), L_{1}(x), L_{2}(x)\right), L_{1}(x), L_{2}(x)\right)$ из $[\Sigma]$, который первые $\tau$ моментов времени работает как константа 0.

Так как $0 \vee S_{0}(y)=S_{0}(y)$ и $0 \vee \bar{y}=\bar{y}$, автоматы $x \vee S_{0}(y), x \vee \bar{y}, 0$ составляют полную систему в $\mathscr{P}_{a}$. Но $\tau$ мы можем выбрать сколь угодно большим, значит, данная система $A$-полна и предложение доказано.

\section{Предложение 5. Система}

$$
\Sigma=\left\{x \vee \bar{y}, x \vee S_{0}(y), h_{4}^{*}, H_{01}, H_{10}, H_{1}, H_{2}, F_{1}, F_{2}, \ldots, F_{k}, T_{1}, T_{2}, \ldots, T_{k}, F, T_{\xi}\right\}
$$

A-полна тогда и только тогда, когда последовательность продукций слова $\xi$ бесконечна.

Доказательство. Достаточность следует из предложения 4.

Докажем необходимость. Пусть последовательность продукций слова $\xi$ конечна и имеет вид $\xi_{1}, \xi_{2}, \ldots, \xi_{s}$. Из предложения 3 следует, что каждый из автоматов в $\Sigma$ сохраняет множество $\widetilde{W}_{\xi}$.

Из предложения 2 следует, что класс всех автоматов, сохраняющих $\widetilde{W}_{\xi}$, замкнут. Но любой автомат, $\tau$-равный константе 0 для $\tau=(s+1) \omega(k+20)$, очевидно, не сохраняет множество $\widetilde{W}_{\xi}$. Так как $[\Sigma]$ сохраняет $\widetilde{W}_{\xi}$, константа 0 не принадлежит $[\Sigma]_{A}$. Тогда $\Sigma$ не $A$-полно. Предложение доказано. 
Доказательство теоремы 3. Так как классы $F_{4}^{4}$ и $F_{8}^{4}$ двойственны, достаточно доказать теорему для $F_{4}^{4}$. Предположим, что теорема неверна. Тогда существует алгоритм, решающий проблему $A$-ПолнОТА $\left(F_{4}^{4}\right)$.

Построим алгоритм, решающий проблему конечности последовательности продукций Поста $\langle D, \rho, \omega\rangle$. По слову $\xi \in D^{*}$ эффективно строится автомат $T_{\xi}$. По предположению, существует алгоритм, решающий проблему $A$-полноты для системы

$$
\Sigma=\left\{x \vee \bar{y}, x \vee S_{0}(y), h_{4}^{*}, H_{01}, H_{10}, H_{1}, H_{2}, F_{1}, F_{2}, \ldots, F_{k}, T_{1}, T_{2}, \ldots, T_{k}, F, T_{\xi}\right\} .
$$

Из предложения 5 следует, что система $\Sigma$ является $A$-полной тогда и только тогда, когда последовательность продукций слова $\xi$ бесконечна.

Таким образом, алгоритм, решающий проблему конечности последовательности продукций Поста $\langle D, \rho, \omega\rangle$, заключается в следующем: по слову $\xi$ строим дефинитный автомат $T_{\xi}$; решаем проблему $A$-полноты для системы

$$
\Sigma=\left\{x \vee \bar{y}, x \vee S_{0}(y), h_{4}^{*}, H_{01}, H_{10}, H_{1}, H_{2}, F_{1}, F_{2}, \ldots, F_{k}, T_{1}, T_{2}, \ldots, T_{k}, F, T_{\xi}\right\} .
$$

Получили противоречие. Теорема доказана.

\section{4. Доказательство утверждений 1 и 3}

Доказательство предложения 1. Пусть $H \in\left\{H_{01}, H_{10}, H_{1}, H_{2}, F_{1}, \ldots, F_{k}, T_{1}, \ldots, T_{k}, F\right.$, $\left.T_{\xi}\right\}, \widetilde{H}$ - отображение, соответствующее $H, \widetilde{H}$ определено на $C$.

Пусть $r_{1} \in C, H\left(r_{1}\right)=\delta^{\prime}, \tilde{H}\left(r_{1}\right)=\delta_{1}$. Покажем, что $\delta^{\prime}=\delta_{1}$. Предположим, что это не так, тогда для какого-то $l_{1}$ выполняется неравенство $\delta^{\prime}\left(l_{1}\right) \neq \delta_{1}\left(l_{1}\right)$. Учитывая определение автомата $H$, получаем, что $\delta^{\prime}\left(l_{1}\right)=0, \delta_{1}\left(l_{1}\right)=1$.

Рассмотрим случай, когда $l_{1}<h(H)$. В этом случае существует $r_{2} \in C$ такое, что ]$\left._{l_{1}} r_{1}=\right]_{l_{1}} r_{2}, \tilde{H}\left(r_{2}\right)=\delta_{2}$, причем $\delta_{2}\left(l_{1}\right)=0 \neq \delta_{1}\left(l_{1}\right)$. Из определения отображений $\widetilde{H}_{01}, \widetilde{H}_{10}, \widetilde{H}_{1}, \widetilde{H}_{2}, \widetilde{F}_{1}, \ldots, \widetilde{F}_{k}, \widetilde{T}_{1}, \ldots, \widetilde{T}_{k}, \widetilde{F}, \widetilde{T}_{\xi}$ следует, что это невозможно.

Теперь рассмотрим случай, когда $l_{1} \geqslant h(H)$. В этом случае существуют $r_{2} \in C$ и $l_{2}$ такие, что $[h(H)]_{l_{1}} r_{1}=[h(H)]_{l_{2}} r_{2}=r, \widetilde{H}\left(r_{2}\right)=\delta_{2}$, причем $\delta_{2}\left(l_{2}\right)=0 \neq \delta_{1}\left(l_{1}\right)$.

Докажем утверждение для автоматов $H_{01}, H_{10}$. Если $r \in\{010,011\}$, то $\delta_{1}\left(l_{1}\right)=\delta_{2}\left(l_{2}\right)=0$, в противном случае $\delta_{2}\left(l_{2}\right)=\delta_{2}\left(l_{2}\right)=1$. Получили противоречие. Другими словами, для любого $i$ выход в момент времени $i$ полностью определяется последними тремя символами и неравенство $\delta_{1}\left(l_{1}\right) \neq \delta_{2}\left(l_{2}\right)$ невозможно.

Докажем утверждение для автоматов $H_{1}$ и $H_{2}$. Пусть $r=\left(\alpha_{1}, \alpha_{2}\right)$. Нетрудно убедиться, что для $H_{1}$ каждое из равенств $\delta_{1}\left(l_{1}\right)=0$ и $\delta_{2}\left(l_{2}\right)=0$ выполняется тогда и только тогда, когда

$$
\alpha_{1}(2(k+20))=0, \quad \alpha_{1}=\left(010001(01)^{k / 2+1}\right)\left((01)^{5} 00010001(01)^{k / 2+1}\right)\left((01)^{5} 01\right)
$$

или

$$
\alpha_{1}=\left(01(01)^{k / 2+1}\right)\left((01)^{5} 00010001(01)^{k / 2+1}\right)\left((01)^{5} 010101\right) .
$$

Аналогично, для $H_{2}$ каждое из равенств $\delta_{1}\left(l_{1}\right)=0$ и $\delta_{2}\left(l_{2}\right)=0$ выполняется тогда и только тогда, когда

$$
\alpha_{2}(2(k+20))=0, \quad \alpha_{2}=\left(000(10)^{k / 2+1}\right)\left((10)^{5} 10100000(10)^{k / 2+1}\right)\left((10)^{5} 10101\right)
$$


или

$$
\alpha_{2}=\left(0(10)^{k / 2+1}\right)\left((10)^{5} 10100000(10)^{k / 2+1}\right)\left((10)^{5} 1010101\right) .
$$

Другими словами, элементы $\delta_{1}\left(l_{1}\right)$ и $\delta_{2}\left(l_{2}\right)$ полностью определяются парой слов $r$. Получили противоречие с равенствами $\delta_{1}\left(l_{1}\right)=1, \delta_{2}\left(l_{2}\right)=0$.

Докажем утверждение для автомата $F_{c}$, где $1 \leqslant c \leqslant k$. Нетрудно убедиться, что для $F_{c}$ каждое из равенств $\delta_{1}\left(l_{1}\right)=0$ и $\delta_{2}\left(l_{2}\right)=0$ выполняется тогда и только тогда, когда $r(2(k+20))=0$ или слово $r$ заканчивается на слово, содержащееся в множестве $\left\{p_{0} \tilde{d}_{j} 1^{c+19}, p_{0} \tilde{d}_{j} 11, p_{0} \tilde{d}_{j} 1(11) 1(11), p_{0} \tilde{d}_{j} 1(11) 1(11) 1(11)\right\}$, то есть, элементы $\delta_{1}\left(l_{1}\right)$ и $\delta_{2}\left(l_{2}\right)$ полностью определяются словом $r$. Получили противоречие.

Пусть $H=T_{c}$, где $1 \leqslant c \leqslant k, r=\left(\alpha_{1}, \alpha_{2}, \alpha_{3}\right)$. $\alpha_{2}$ всегда заканчивается на слово вида $e_{1} \gamma$, где $\left.\gamma=\right]_{m}\left(e_{1}\right), 0 \leqslant m<(k+20)$. Нетрудно убедиться, что каждое из равенств $\delta_{1}\left(l_{1}\right)=0$ и $\delta_{2}\left(l_{2}\right)=0$ выполняется только в следующих случаях:

(1) $\alpha_{1}$ заканчивается на 0 и $m \notin\{2,8\}$;

(2) $m=3$ и $\alpha_{1}$ заканчивается на $p_{3} \tilde{g}_{0} p_{0} \tilde{g}_{1} \ldots p_{0} \tilde{g}_{s} 101$, где $s<\left|R_{c}\right|, g_{i} \in D$ для любого $i$;

(3) $m=2$ и $\alpha_{1}$ заканчивается на $p_{0} \tilde{g}_{1} \ldots p_{0} \tilde{g}_{s} 10$, где $s \geqslant\left|R_{c}\right|, g_{i} \in D$ для любого $i$;

(4) $m=8$ и $\alpha_{1}$ заканчивается на $p_{2} \tilde{g}_{1} p_{3} \tilde{g}_{2} \ldots p_{3} \tilde{g}_{\omega} 1(\varepsilon) 1(10) 11$, где $\varepsilon \in\{01,10\}, g_{i} \in D$ для любого $i$;

(5) $m=5$ и $\alpha_{1}$ заканчивается на $p_{2} \tilde{g}_{1} p_{3} \tilde{g}_{2} \ldots p_{3} \tilde{g}_{s} 1(\varepsilon) 11$, где $s \leqslant \omega, \varepsilon \in\{01,10\}$, $g_{i} \in D$ для любого $i$.

Таким образом, элементы $\delta_{1}\left(l_{1}\right)$ и $\delta_{2}\left(l_{2}\right)$ полностью определяются тройкой $r$. Получили противоречие с неравенством $\delta_{1}\left(l_{1}\right) \neq \delta_{2}\left(l_{2}\right)$.

Пусть $H=F$. Из определения следует, что $\delta_{1}=\delta_{2}=0^{\infty}$. Значит $\delta_{2}\left(l_{2}\right)=0$. Получили противоречие.

Для $H=T_{\xi}$ каждое из равенств $\delta_{1}\left(l_{1}\right)=0$ и $\delta_{2}\left(l_{2}\right)=0$ выполняется тогда и только тогда, когда $r$ заканчивается на 0 . Получили противоречие с неравенством $\delta_{1}\left(l_{1}\right) \neq \delta_{2}\left(l_{2}\right)$. Предложение доказано.

Лемма 1. Если последовательность продукиий слова छ конечна, то каждый из автоматов $x \vee \bar{y}, x \vee S_{0}(y), h_{4}^{*}$, сохраняет множество $\widetilde{W}_{\xi}$.

Доказательство. Докажем лемму для автоматов $x \vee \bar{y}$ и $x \vee S_{0}(y)$. Если на первый вход любого из этих автоматов в какой-то момент времени подавалась 1, то и на выходе в этот момент времени будет 1 . Поэтому, если на первый вход подавалось сверхслово из $\widetilde{W}_{\xi}$, то и на выходе будет сверхслово из $\widetilde{W}_{\xi}$.

Докажем лемму для автомата $h_{4}^{*}\left(x_{1}, x_{2}, x_{3}, x_{4}, x_{5}\right)$. Предположим, что это не так. Пусть $\alpha_{1}, \alpha_{2}, \alpha_{3}, \alpha_{4}, \alpha_{5} \in \widetilde{W}_{\xi}$, а $h_{4}^{*}\left(\alpha_{1}, \alpha_{2}, \alpha_{3}, \alpha_{4}, \alpha_{5}\right)=\beta \notin \widetilde{W}_{\xi}$. Значит, для какой-то тройки $\left(n_{1}, n_{2}, n_{3}\right) \in W_{\xi}$ верны равенства $\beta\left(n_{1}\right)=\beta\left(n_{2}\right)=\beta\left(n_{3}\right)=\beta\left(n_{4}(\xi)\right)=0$. Так как на вход в $h_{4}^{*}$ подается 5 сверхслов и для каждого сверхслова $\alpha_{i}$ найдется $j \in\{1,2,3,4\}$ такое, что $\alpha_{i}\left(n_{j}\right)=1$, для каких-то $j, m, l, m \neq l$ выполняются равенства $\alpha_{m}\left(n_{j}\right)=\alpha_{l}\left(n_{j}\right)=1$. Но в этом случае $\beta\left(n_{j}\right)=1$. Получили противоречие. Лемма доказана. 
Лемма 2. Для любых $H \in\left\{H_{01}, H_{10}, F_{1}, F_{2}, \ldots, F_{k}\right\}, \beta=H(\alpha), \alpha \in E, i \in \mathbf{N}$ выполняется равенство

$$
\beta(i) \vee \beta(i+1)=1 .
$$

Доказательство. Для $i<h(H)$ утверждение очевидно. Докажем его для $i \geqslant h(H)$. Предположим, что это не так и $\beta(i)=\beta(i+1)=0$. Пусть $H \in\left\{H_{01}, H_{10}\right\}$. Так как $\beta(i+1)=0$, справедливы равенства $\alpha(i-1)=0, \alpha(i)=1$. Но тогда $\beta(i)=1$. Получили противоречие.

Теперь докажем лемму для $F_{c}$, где $1 \leqslant c \leqslant k$. Как было указано выше, если $\beta(i)=0$, то $\alpha(i)=0$ или слово $]_{i} \alpha$ заканчивается на слово из множества

$$
R=\left\{p_{0} \tilde{d}_{j} 1^{c+13}, p_{0} \tilde{d}_{j} 11, p_{0} \tilde{d}_{j} 1(11) 1(11), p_{0} \tilde{d}_{j} 1(11) 1(11) 1(11)\right\} .
$$

Если $\alpha(i)=0$ и $\alpha(i+1)=0$, то $\beta(i+1)=1$, так как в $C_{F}$ нет сверхслов, в которых в каком-то месте идут подряд 2 нуля. Если $\alpha(i)=0$, а $\alpha(i+1)=1$, то $\beta(i+1)=1$, так как в $R$ нет слов, заканчивающихся на 01 . Пусть $\alpha(i)=1$, а $]_{i} \alpha$ заканчивается на слово из множества $R$. Легко проверить, что в любом сверхслове из $C_{F}$ после слова из $R$ обязательно идет 1 . Значит $\alpha(i+1)=1$. Но в этом случае $]_{i+1} \alpha$ не может заканчиваться на слово из $R$. Получили противоречие. Лемма доказана.

Следствие 1. Если последовательность продукций слова $\xi$ конечна, то каждый из автоматов $H_{01}, H_{10}, F_{1}, \ldots, F_{k}$ сохраняет множество $\widetilde{W}_{\xi}$.

Доказательство. Как было замечено ранее, для любой тройки $\left(n_{1}, n_{2}, n_{3}\right) \in W_{\xi}$ выполняется равенство $n_{1}+1=n_{2}$. Из леммы 2 следует, что если $H \in\left\{H_{01}, H_{10}, F_{1}, \ldots, F_{k}\right\}$, то для $\beta=H(\alpha)$ справедливо равенство $\beta\left(n_{1}\right) \vee \beta\left(n_{2}\right)=1$. Значит,

$$
\beta\left(n_{1}\right) \vee \beta\left(n_{2}\right) \vee \beta\left(n_{3}\right) \vee \beta\left(n_{4}(\xi)\right)=1,
$$

и следствие доказано.

Лемма 3. Если последовательность продукций слова $\xi$ конечна, то автоматы $\mathrm{H}_{1}, \mathrm{H}_{2}$ сохраняют множество $\widetilde{W}_{\xi}$.

Доказательство. Идея доказательства заключается в следующем. Если сверхслово принадлежит $\widetilde{W}_{\xi}$ и на $n_{4}(\xi)$-м месте в нем стоит 0 , то слово 0001000 может в нем идти только начиная с позиции $(k+20) m+11$, а слово $00000-$ с позиции $(k+20) m+14$, где $m<b_{s}$. Чтобы на выходе в момент времени $n_{4}(\xi)$ был 0, необходимо, чтобы либо на первый вход, либо на второй вход в момент времени $n_{4}(\xi)$ подавался 0 . С помощью этого мы всегда можем определить остаток от деления номера позиции на $k+20$, и обеспечить, чтобы данные автоматы сохраняли множество $\widetilde{W}_{\xi}$.

Перейдем к доказательству. Докажем сначала утверждение для автомата $H_{1}$. Предположим, что лемма неверна, то есть $\alpha_{1}, \alpha_{2} \in \widetilde{W}_{\xi}$, а $H_{1}\left(\alpha_{1}, \alpha_{2}\right)=\beta \notin \widetilde{W}_{\xi}$. Значит, найдется тройка $\left(n_{1}, n_{2}, n_{3}\right) \in W_{\xi}$ такая, что

$$
\beta\left(n_{1}\right)=\beta\left(n_{2}\right)=\beta\left(n_{3}\right)=\beta\left(n_{4}(\xi)\right)=0 .
$$

Справедливо равенство $\beta\left(n_{4}(\xi)\right)=0$, поэтому либо $\alpha_{1}\left(n_{4}(\xi)\right)=0$, либо $\alpha_{2}\left(n_{4}(\xi)\right)=0$. По определению, тройка $\left(n_{1}, n_{2}, n_{3}\right)$ имеет вид $n_{1}=(k+20) n+q_{1}, n_{2}=(k+20) n+q_{2}$, где $0 \leqslant q_{1}, q_{2} \leqslant(k+20), q_{2}-q_{1}=1$. Если $n=0$, то утверждение очевидно, так как автомат $H_{1}$ в моменты времени $2,4,6,8,10,14,18,20,22, \ldots,(k+20)$ возвращает единицу. 
Пусть $n \geqslant 1$. Если $\alpha_{1}\left(n_{4}(\xi)\right)=1$, то $\alpha_{2}\left(n_{4}(\xi)\right)=0$. Так как $\alpha_{1}\left(n_{4}(\xi)\right)=1$ и $\beta\left(n_{4}(\xi)\right)=0$, слово $]_{n_{4}(\xi)} \alpha_{2}$ заканчивается на $\left((10)^{5} 10100000(10)^{k / 2+1}\right)\left((10)^{5} 10\right)$ или на $\left((10)^{5} 10100000(10)^{k / 2+1}\right)\left((10)^{5} 101010\right)$. Но в этом случае слово 00000 идет в сверхслове $\alpha_{2}$, начиная с позиции $(k+20) m+8$ или $(k+20) m+4$, что невозможно.

Теперь рассмотрим случай, когда $\alpha_{1}\left(n_{4}(\xi)\right)=0$. Поскольку $\beta\left(n_{4}(\xi)\right)=0$, $\alpha_{1} \in \widetilde{W}_{\xi}, n_{4}(\xi)=b_{s}(k+20)+6$, а слово 0001000 может идти в $\alpha_{1}$ только начиная с позиции $(k+20) m+11$, слово $]_{n_{4}(\xi)} \alpha_{1}$, очевидно, должно заканчиваться на слово $00010001(01)^{k / 2+1} 010101$. Но мы предположили, что $\alpha_{1}\left(n_{4}(\xi)\right)=0$, получили противоречие.

Теперь докажем утверждение для автомата $H_{2}$. Предположим, что лемма неверна, то есть, $\alpha_{1}, \alpha_{2} \in \widetilde{W}_{\xi}$, а $H_{2}\left(\alpha_{1}, \alpha_{2}\right)=\beta \notin \widetilde{W}_{\xi}$. Значит, есть тройка $\left(n_{1}, n_{2}, n_{3}\right) \in W_{\xi}$ такая, что $\beta\left(n_{1}\right)=\beta\left(n_{2}\right)=\beta\left(n_{3}\right)=\beta\left(n_{4}(\xi)\right)=0$. Так как $\beta\left(n_{4}(\xi)\right)=0$, верно либо $\alpha_{1}\left(n_{4}(\xi)\right)=0$, либо $\alpha_{2}\left(n_{4}(\xi)\right)=0$. По определению, тройка $\left(n_{1}, n_{2}, n_{3}\right)$ имеет вид $n_{1}=(k+20) n+q_{1}$, $n_{2}=(k+20) n+q_{2}$, где $0 \leqslant q_{1}, q_{2} \leqslant(k+20), q_{2}-q_{1}=1$. Если $n=0$, то утверждение очевидно, так как автомат $H_{2}$ в моменты времени $1,3,5,7,9,11,13,19,21,23, \ldots,(k+19)$ возвращает единицу.

Пусть $n \geqslant 1$. Рассмотрим случай, когда $\alpha_{1}\left(n_{4}(\xi)\right)=0$. Так как $\beta\left(n_{4}(\xi)\right)=0$, $\alpha_{1} \in \widetilde{W}_{\xi}, n_{4}(\xi)=b_{s}(k+20)+6$, а слово 0001000 может идти в $\alpha_{1}$ только начиная с позиции $(k+20) m+11$, слово $]_{n_{4}(\xi)} \alpha_{1}$, очевидно, должно заканчиваться на слово $00010001(01)^{k / 2+1} 010101$. Но мы предположили, что $\alpha_{1}\left(n_{4}(\xi)\right)=0$. Получили противоречие.

Теперь рассмотрим случай, когда $\alpha_{2}\left(n_{4}(\xi)\right)=0$. Поскольку $n \geqslant 1$,

$$
\beta_{1}\left(n_{2}-1\right)=\beta_{1}\left(n_{2}\right)=\beta\left(n_{4}(\xi)\right)=0,
$$

слово $]_{n_{2}} \alpha_{2}$ заканчивается на слово $10100000(10)^{k / 2+1}(10)^{5} \gamma$, где $|\gamma| \in\{5,6,7,8\}$. Иными словами, если автомат $H_{2}$ на выходе дает подряд два нуля, то около $k+20$ букв назад на вход подавалось слово 00000. Но слово 00000 может встретиться только на позиции $(k+20) m+14$. Тогда $q_{2} \in\{15,16,17,18\}$. Но $q_{2}-q_{1}=1$, значит, тройки $\left(n_{1}, n_{2}, n_{3}\right)$ нет в множестве $W_{\xi}$. Получили противоречие. Значит, автомат $H_{2}$ сохраняет $\widetilde{W}_{\xi}$.

Лемма 4. Если последовательность продукиий слова $\xi$ конечна, то автомат $F$ сохраняет множество $\widetilde{W}_{\xi}$.

Доказательство. Предположим, что это не так, то есть, что $\alpha_{1}, \alpha_{2}, \alpha_{3} \in \widetilde{W}_{\xi}$, но $F\left(\alpha_{1}, \alpha_{2}, \alpha_{3}\right)=\beta \notin \widetilde{W}_{\xi}$. Значит, найдется тройка $\left(n_{1}, n_{2}, n_{3}\right) \in W_{\xi}$ такая, что

$$
\beta\left(n_{1}\right)=\beta\left(n_{2}\right)=\beta\left(n_{3}\right)=\beta\left(n_{4}(\xi)\right)=0 .
$$

Так как $\beta\left(n_{4}(\xi)\right)=0$, либо $\alpha_{2}\left(n_{4}(\xi)\right)=0$, либо $\alpha_{3}\left(n_{4}(\xi)\right)=0$.

Идея доказательства заключается в следующем. С помощью $\alpha_{2}$ или $\alpha_{3}$ мы в каждый момент времени можем определить остаток от деления момента времени на $(k+20)$. Для любого $n$ автомат $F$ действует таким образом, что если в момент времени $(k+20) n+6$ на первый вход подается 1 , то и на выходе в момент времени $(k+20) n+6$ будет 1 . И если в момент времени $(k+20) n+5$ на первый вход подается 1 , то опять же на выходе в момент времени $(k+20) n+6$ будет 1 .

Рассмотрим случай, когда $\alpha_{2}\left(n_{4}(\xi)\right)=0$. Поскольку $\beta\left(n_{4}(\xi)\right)=0, \alpha_{2} \in \widetilde{W}_{\xi}$, $n_{4}(\xi)=b_{s}(k+20)+6$, а слово 0001000 может идти в $\alpha_{2}$ только начиная с позиции $(k+20) m+11$, слово $]_{n_{4}(\xi)} \alpha_{2}$ должно заканчиваться на слово $00010001(01)^{k / 2+1} 010101$. Но мы предположили, что $\alpha_{2}\left(n_{4}(\xi)\right)=0$, получили противоречие. 
Рассмотрим случай, когда $\alpha_{3}\left(n_{4}(\xi)\right)=0$. Поскольку $\beta\left(n_{4}(\xi)\right)=0, \alpha_{3} \in \widetilde{W}_{\xi}$, а $n_{4}(\xi)=b_{s}(k+20)+6$, слово $]_{n_{4}(\xi)} \alpha_{3}$ заканчивается на слово $10100000(10)^{k / 2+1} 101010$. Так как $\alpha_{1} \in \widetilde{W}_{\xi}$, мы видим, что $\alpha_{1}\left(n_{4}(\xi)-1\right) \vee \alpha_{1}\left(n_{4}(\xi)\right)=1$. Но в этом случае из определения автомата $F$ получаем, что $\beta\left(n_{4}(\xi)\right)=1$. Получили противоречие. Значит, автомат $F$ сохраняет множество $\widetilde{W}_{\xi}$.

Лемма 5. Если последовательность продукций слова $\xi$ конечна, то автомат $T_{c}$, где $1 \leqslant c \leqslant k$, сохраняет множество $\widetilde{W}_{\xi}$.

Доказательство. Предположим, что это не так, то есть, что $\alpha_{1}, \alpha_{2}, \alpha_{3} \in \widetilde{W}_{\xi}$, но $T_{c}\left(\alpha_{1}, \alpha_{2}, \alpha_{3}\right)=\beta \notin \widetilde{W}_{\xi}$. Значит, найдется тройка $\left(n_{1}, n_{2}, n_{3}\right) \in W_{\xi}$ такая, что

$$
\beta\left(n_{1}\right)=\beta\left(n_{2}\right)=\beta\left(n_{3}\right)=\beta\left(n_{4}(\xi)\right)=0 .
$$

Так как $\beta\left(n_{4}(\xi)\right)=0$, либо $\alpha_{2}\left(n_{4}(\xi)\right)=0$, либо $\alpha_{3}\left(n_{4}(\xi)\right)=0$. Пусть $n_{1}=(k+20) n+q_{1}$, $n_{2}=(k+20) n+q_{2}$, где $0 \leqslant q_{1}, q_{2} \leqslant(k+20), q_{2}-q_{1}=1$.

Рассмотрим случай, когда $\alpha_{2}\left(n_{4}(\xi)\right)=0$. Поскольку $\beta\left(n_{4}(\xi)\right)=0, \alpha_{2} \in \widetilde{W}_{\xi}$, $n_{4}(\xi)=b_{s}(k+20)+6$, а слово 0001000 может идти в $\alpha_{2}$, только начиная с позиции $(k+20) m+11$, слово $]_{n_{4}(\xi)} \alpha_{2}$ должно заканчиваться словом $00010001(01)^{k / 2+1}(01)^{3}$. Но мы предполагали, что $\alpha_{2}\left(n_{4}(\xi)\right)=0$. Получили противоречие.

Теперь рассмотрим случай, когда $\alpha_{3}\left(n_{4}(\xi)\right)=0$. Поскольку $\beta\left(n_{4}(\xi)\right)=0$, $\alpha_{3} \in \widetilde{W}_{\xi}$, а $n_{4}(\xi)=b_{s}(k+20)+6$, слово $]_{n_{4}(\xi)} \alpha_{3}$, очевидно, заканчивается на слово $10100000(10)^{k / 2+1}(10)^{3}$. Значит, $\alpha_{1}\left(n_{4}(\xi)\right)=0$. На выходе в моменты времени $n_{1}$ и $n_{2}=n_{1}+1$ автомат $T_{c}$ выдал 0 , поэтому $]_{n_{2}} \alpha_{3}$ может заканчиваться только на $10100000(10)^{k / 2+1}(10)^{3}$ или на $00010001(10)^{k / 2+1}(10)^{4} 1$. Значит, $q_{1}=5$, либо $q_{1}=8$.

Вспомним обозначения из определения множества $\widetilde{W}_{\xi}$. Пусть последовательность продукций слова $\xi$ имеет вид $\xi_{1}, \xi_{2}, \ldots, \xi_{s}$; для любого $t$ слово $\xi_{t}$ начинается с буквы $d_{i_{t}}$. Рассмотрим последовательности

$$
a_{i}=(i-1) \omega, \quad b_{i}=a_{i}+\left|\xi_{i}\right|
$$

Тогда

$$
n_{4}(\xi)=b_{s}(k+20)+6 .
$$

Положим

$$
\xi_{1} R_{i_{1}} R_{i_{2}} \ldots R_{i_{s-1}}=g_{1} g_{2} \ldots g_{l}
$$

Пусть для $1 \leqslant i \leqslant s$

$$
\zeta_{i}=p_{1} \tilde{g}_{1} p_{1} \tilde{g}_{2} \ldots p_{1} \tilde{g}_{a_{i}} p_{2} \tilde{g}_{a_{i}+1} p_{3} \tilde{g}_{a_{i}+2} \ldots p_{3} \tilde{g}_{b_{i}} p_{0} \tilde{g}_{b_{i}+1} \text {. }
$$

Если $q_{1}=5$, то $n_{1}=n_{4}(\xi)-1, n_{2}=n_{3}=n_{4}(\xi)$. Так как $\alpha_{1} \in \widetilde{W}_{\xi}$, мы видим, что $\alpha_{1}\left(n_{4}(\xi)-1\right) \vee \alpha_{1}\left(n_{4}(\xi)\right)=1$. Из определения автомата $T_{c}$ следует, что $]_{n_{4}(\xi)} \alpha_{1}$ заканчивается на $p_{2} \tilde{d}_{c} p_{3} \tilde{v}_{1} p_{3} \tilde{v}_{2} \ldots p_{3} \tilde{v}_{m-1} 1(\varepsilon) 1(10)$, где $v_{i} \in D$ для любого $i, m \leqslant \omega$, $\varepsilon \in\{01,10\}$. При этом $\varepsilon$ равняется 01 тогда и только тогда, когда $m=\omega$.

Так как $\alpha_{1} \in \widetilde{W}_{\xi}$ и

$$
\alpha_{1}\left(\left(b_{s}-m\right)(k+20)+8\right)=\alpha_{1}\left(\left(b_{s}-m\right)(k+20)+9\right)=\alpha_{1}\left(n_{4}(\xi)\right)=0,
$$


верно соотношение $m=\left|\xi_{s}\right|<\omega$. Но тройка $\left((k+20) a_{s}+8,(k+20) a_{s}+9,(k+20) b_{s}+3\right)$ принадлежит $W_{\xi}$, поэтому $\alpha_{1}\left((k+20) b_{s}+3\right)=1$ и $\varepsilon=01$. Получили противоречие, так как $\varepsilon$ не может равняться 01 , если $m<\omega$.

Таким образом, осталось рассмотреть случай $\alpha_{1}\left(n_{4}(\xi)\right)=0, q_{1}=8$. Из определения автомата следует, что $]_{n_{2}} \alpha_{1}$ заканчивается на $p_{2} \tilde{d}_{c} p_{3} \tilde{v}_{1} p_{3} \tilde{v}_{2} \ldots p_{3} \tilde{v}_{\omega-1} 1(\varepsilon) 1(10) 1(10)$, где $v_{i} \in D$ для любого $i, \varepsilon \in\{01,10\}$. В этом случае

$$
\alpha_{1}((n-\omega)(k+20)+8)=\alpha_{1}((n-\omega)(k+20)+9)=\alpha_{1}\left(n_{4}(\xi)\right)=0 .
$$

Значит, для какого-то $m \in \mathbf{N}$ выполняются равенства

$$
n=(m-1) \omega=a_{m} .
$$

При этом

$$
\zeta_{m}\left(n_{3}\right)=1, \quad n_{3} \leqslant b_{m}(k+20)+4 .
$$

Из определения множества $\widetilde{W}_{\xi}$ получим, что для $i \leqslant b_{m-1}(k+20)+4$ справедливо неравенство $\alpha_{1}(i) \geqslant \zeta_{m-1}(i)$. Порождающая функция $T_{c}^{h\left(T_{c}\right)}$ обладает таким свойством, что если в слове $\left[h\left(T_{c}\right)\right]_{i} \zeta_{m-1}$, подаваемом на первый вход, поменять некоторые символы с 0 на 1, то значение либо не изменится, либо будет равно 1. Значит, для любого $i \leqslant b_{m-1}(k+20)+4$ выполняется неравенство $\beta(i) \geqslant \zeta_{m}(i)$. А значит, для $n_{3} \leqslant b_{m-1}(k+20)+4$ лемма доказана.

Теперь пусть

$$
b_{m-1}(k+20)+4<n_{3} \leqslant b_{m}(k+20)+4 .
$$

Из условия следует, что для $i \leqslant\left(b_{m-1}+1\right)(k+20)+4$ выполняется неравенство

$$
\alpha_{1}(i) \geqslant \zeta_{m-1}(i)
$$

Пусть

$$
\gamma=p_{3} \tilde{g}_{b_{m-1}} 1(01) 1 .
$$

Так как $\zeta_{m-1}(i)$ заканчивается на $p_{3} \tilde{g}_{b_{m-1}} p_{0} \tilde{g}_{b_{m-1}+1}$, а высота автомата $T_{c}$ равна $\left(\omega+\left|R_{c}\right|+2\right)(k+20)$, на первый вход порождающей функции $T_{c}^{h\left(T_{c}\right)}$ в момент времени $n_{3}$ поступит слово, заканчивающееся на $\gamma_{1} \gamma_{2}$, где $\left|\gamma_{1}\right|=|\gamma|$ и для любого $i$ выполняется неравенство $\gamma_{1}(i) \geqslant \gamma(i)$. Из определения автомата следует, что в этом случае $\beta\left(n_{3}\right) \geqslant \zeta_{m}\left(n_{3}\right)$. Значит, $\beta\left(n_{3}\right)=\zeta_{m}\left(n_{3}\right)=1$. Получили противоречие. Лемма доказана.

Лемма 6. Если последовательность продукиий слова $\xi$ конечна, то автомат $T_{\xi}$ сохраняет множество $\widetilde{W}_{\xi}$.

Доказательство. Предположим, что это не так, то есть, $\alpha \in \widetilde{W}_{\xi}$, а $T_{\xi}(\alpha)=\beta \notin \widetilde{W}_{\xi}$. Значит, найдется тройка $\left(n_{1}, n_{2}, n_{3}\right) \in W_{\xi}$ такая, что

$$
\beta\left(n_{1}\right)=\beta\left(n_{2}\right)=\beta\left(n_{3}\right)=\beta\left(n_{4}(\xi)\right)=0 .
$$

Пусть $\xi=g_{1} g_{2} \ldots g_{l}$. Автомат $T_{\xi}$ работает таким образом, что в момент времени $t>(|\xi|+2)(k+20)$ он возвращает либо 1 , либо то, что подавалось на вход в момент $t$. Если $\alpha(t-1)=\alpha(t)=0$, то, очевидно, $\beta(t)=1$. Таким образом, начиная $\mathrm{c}$ 
момента времени $(|\xi|+2)(k+20)$ на выходе не может появиться два нуля подряд. В первые $(|\xi|+2)(k+20)$ моментов времени он выводит либо по очереди буквы сверхслова

$$
\alpha_{0}=p_{2} \tilde{g}_{1} p_{3} \tilde{g}_{2} \ldots p_{3} \tilde{g}_{l} p_{0} \tilde{g}_{l+1} p_{0} \tilde{g}_{l+2} p_{0} \tilde{g}_{l+3} \ldots,
$$

либо 1. Таким образом, два нуля подряд могут появиться только на 5 и 6, или 8 и 9 позиции. По определению, тройка $\left(8,9, n_{3}\right)$ принадлежит $W_{\xi}$ тогда и только тогда, когда $n_{3} \leqslant|\xi|(k+20)+4$. Значит, из того, что $\alpha_{0} \in \widetilde{W}_{\xi}$ следует, что $\beta \in \widetilde{W}_{\xi}$. Получили противоречие. Значит, $T_{\xi}$ сохраняет множество $\widetilde{W}_{\xi}$.

Из лемм 1-6, а также следствия 1 следует предложение 3.

\section{Список литературы}

1. Post E., Two-valued iterative systems of mathematical logic. Princeton Univ. Press, Princeton, 1941.

2. Яблонский С. В., Гаврилов Г. П., Кудрявцев В. Б., Функиии алгебры логики и классы Поста. Наука, Москва, 1966.

3. Кратко М. И., Алгоритмическая неразрешимость проблемы распознавания полноты для конечных автоматов. Доклады АН СССР (1964) 155, 35-37.

4. Буевич В. А., Об алгоритмической неразрешимости распознавания $A$-полноты для о.д.-функций. Математические заметки (1972) 12, №6, 687-697.

5. Бабин Д. Н., Разрешимый случай задачи о полноте автоматных функций. Дискретная математика (1992) 4, №4, 41-56.

6. Буевич В. А., Условия А-полноты для автоматов. Изд-во МГУ, Москва, 1986.

7. Бабин Д. Н., О классификации автоматных базисов Поста по разрешимости свойств полноты и $A$-полноты. Доклады РАН (1999) 367, №4, 439-441.

8. Буевич В. А., Клиндухова Т. Э., Об алгоритмической неразрешимости задач об $A$-полноте и полноте для дефинитных ограниченно-детерминированных функций. Матем. вопросы кибернетики (2001), № 10.

9. Жук Д. Н., Присмотров Ю. Н., О проблеме полноты в классе автоматов без обратной связи. Интеллектуальные системы (2007) 11, 439-472.

10. Жук Д. Н., О неразрешимости проблемы полноты для дефинитных автоматов. Интеллектуальные системы (2008) 12, 211-228.

11. Жук Д. Н., Разрешимые случаи задачи об $A$-полноте для дефинитных автоматов. Интеллектуальные системы (2009) 13, 273-312.

12. Кудрявцев В. Б., Алешин С. В., Подколзин А. С.Введение в теорию автоматов. Наука, Москва, 1985.

13. Мальцев А. И., Алгоритмы и рекурсивные функции. Наука, Москва, 1986.

Статья поступила 3.02.2010. 